\title{
Construction of Interval Wavelet Based on Restricted Variational Principle and Its Application for Solving Differential Equations
}

\author{
Shu-Li Mei, ${ }^{1}$ Hong-Liang Lv, ${ }^{1}$ and Qin $\mathrm{Ma}^{1}$ \\ ${ }^{1}$ College of Information and Electrical Engineering, China Agricultural University, P.O. Box 053, \\ 17 Qinghua Donglu Road, Beijing 100083, China
}

Correspondence should be addressed to Shu-Li Mei, meishuli@163.com

Received 10 December 2007; Revised 25 February 2008; Accepted 31 March 2008

Recommended by Giuseppe Rega

Based on restricted variational principle, a novel method for interval wavelet construction is proposed. For the excellent local property of quasi-Shannon wavelet, its interval wavelet is constructed, and then applied to solve ordinary differential equations. Parameter choices for the interval wavelet method are discussed and its numerical performance is demonstrated.

Copyright (C) 2008 Shu-Li Mei et al. This is an open access article distributed under the Creative Commons Attribution License, which permits unrestricted use, distribution, and reproduction in any medium, provided the original work is properly cited.

\section{Introduction}

Since the definition domain of wavelet transformation is an infinite interval, the boundary effect would occur when applied for resolving the engineering problems with bounded interval, for example, ordinary differential equations (ODEs). Consequently, it will decrease the precision and computational efficiency of the solution. Nevertheless, the boundary effect can be eliminated effectively by constructing an interval wavelet using numerical methods.

There are several ways available to construct an interval wavelet. In general, the construction method is relative to the wavelet function, that is, different interval wavelet has different construction method. A simple solution is the even 2-periodical extension $\tilde{f}$ of function $f:[0,1] \rightarrow \mathbb{R}$, which is usually used in image analysis. Unfortunately, this extension generally produces discontinuities at the integers that are indicated by the large wavelet coefficients near the endpoints 0 and 1 . Thus, the constructed wavelet cannot exactly analyze the boundary behavior of a given function. To solve this problem, the popular method is based on special boundary and interior scaling functions as well as wavelets to reduce the numerical problem at the boundaries $[1,2]$. 
The aim of this paper is to introduce a general construction method of interval wavelet based on the restricted variational principle. As an example, the quasi-Shannon wavelet is firstly introduced in brief. Then, its corresponding interval wavelet is constructed in detail based on the restricted variational principle [3]. After that, the wavelet collocation method is applied to obtain the interval wavelet discrete formulation of ODEs. The performance of the quasi-Shannon interval wavelet is illustrated by comparing the numerical results of Shannon, quasi-Shannon, and Shannon interval wavelets on a convection equation.

\section{Quasi-shannon wavelet}

The Shannon scaling function is smooth, this means that the function and all of its derivatives exist and are continuous. But the Shannon function does not have compact support. In order to improve the localized and asymptotic behavior of the Shannon scaling function, Wei [4] introduced a regularization factor $R(x)$ as follows:

$$
w(x)=\phi(x) R(x), \quad \phi(x)=\frac{\sin (\pi x)}{\pi x} .
$$

The advantage of the regularized Shannon scaling function is that its Fourier transformation is continuous [4], resulting in an excellent local property. A common and important regularization factor $R(x)$ proposed by Wei [4] is the Gaussian function

$$
R_{\sigma}(x)=\exp \left(-\frac{x^{2}}{2 \sigma^{2}}\right), \quad \sigma>0,
$$

where $\sigma$ is the width parameter (or called window size). In practice, the best results are usually obtained, when $\sigma=r \Delta$ [4] ( $r$ is a parameter chosen in computations, $\Delta$ is the size of cell in discrete mesh). Substituting (2.2) into (2.1), the Gaussian regularized orthogonal sampling scaling function can be obtained as following:

$$
w(x)=\frac{\sin (\pi x)}{\pi x} \exp \left(-\frac{x^{2}}{2 \sigma^{2}}\right), \quad \sigma>0 .
$$

The corresponding discrete formula is

$$
w\left(x-x_{i}\right)=\frac{\sin (\pi / \Delta)\left(x-x_{i}\right)}{(\pi / \Delta)\left(x-x_{i}\right)} \exp \left(-\frac{\left(x-x_{n}\right)^{2}}{2 \sigma^{2}}\right) .
$$

\section{Construction of interval wavelet based on restricted variational principle}

\subsection{Restricted variational principle}

Defining a scalar fonctionelle $\prod$ of $u$ as

$$
\prod=\int_{\Omega} F\left(u, \frac{\partial u}{\partial x}, \ldots\right) \mathrm{d} \Omega+\int_{\Gamma} E\left(u, \frac{\partial u}{\partial x}, \ldots\right) \mathrm{d} \Gamma
$$


where $u$ is an unknown function, both $F$ and $E$ are specific operators, $\Omega$ is the definition domain, and $\Gamma$ is the boundary of $\Omega$. The solution of a continuous medium problem is a stagnation point of the functional equation $\Pi$, that is, its variation is equal to zero

$$
\delta \prod=0 \text {. }
$$

The method described above for solving a continuous medium problem is called restricted variational principle [3].

The unknown function $u$ could be approximately expressed as

$$
u \approx \tilde{u}=\sum_{i=1}^{n} N_{i} a_{i}=\mathbf{N a},
$$

where $a_{i}$ is the parameters to be determined and $N_{i}$ is the trial function.

If the unknown function $u$ is restricted by additional condition $C(u)=0$ in definition domain $\Omega$, a revised fonctionelle $\Pi^{*}$ could be constructed as follows:

$$
\prod^{*}=\prod+\int_{\Omega} \lambda^{T} C(u) \mathrm{d} \Omega,
$$

where $\lambda$ is called Lagrange multiplier, which is a function vector with independent coordinates in $\Omega$. The functions $u$ and $\lambda$ are two unknown variables within the revised fonctionelle. Similarly as (3.3), $\lambda$ could be approximately expressed by trial function $\widetilde{N}_{i}$ as

$$
\tilde{\imath}=\sum \widetilde{N}_{i} b_{i}=\tilde{\mathbf{N}} \mathbf{b}
$$

Let the variation of the revised fonctionelle equal to zero, and a system of equations could be obtained as follows:

$$
\frac{\delta \Pi^{*}}{\delta \mathbf{c}}=\left\{\begin{array}{c}
\frac{\delta \Pi^{*}}{\delta \mathbf{a}} \\
\frac{\delta \prod^{*}}{\delta \mathbf{b}}
\end{array}\right\}=0, \quad \mathbf{c}=\left\{\begin{array}{l}
\mathbf{a} \\
\mathbf{b}
\end{array}\right\} .
$$

The values of $\mathbf{a}$ and $\mathbf{b}$ could be obtained by solving the above system of equations.

Let the Euler equation of the fonctionelle $\Pi$ is

$$
A(u)=0,
$$

and the additional condition is

$$
C(u)=L_{1}(u)+C_{1}=0 .
$$

Substituting (3.5), (3.7), and (3.8) into (3.4), the following function could be obtained:

$$
\delta \prod^{*}=\delta \mathbf{a}^{T} \int_{\Omega} \mathbf{N}^{T} A(\tilde{u}) \mathrm{d} \Omega+\delta \mathbf{b}^{T} \int_{\Omega} \tilde{\mathbf{N}}\left[L_{1}(\tilde{u})+C_{1}\right] \mathrm{d} \Omega+\delta \mathbf{a}^{T} \int_{\Omega} L_{1}^{T}(\mathbf{N}) \tilde{\lambda} \mathrm{d} \Omega=0 .
$$

Since (3.9) is satisfied for all variations of $\delta \mathbf{a}$ and $\delta \mathbf{b}$, the following two equations can be derived:

$$
\begin{gathered}
\int_{\Omega} \mathbf{N}^{T} A(\tilde{u}) \mathrm{d} \Omega+\int_{\Omega} L_{1}^{T}(\mathbf{N}) \tilde{\lambda} \mathrm{d} \Omega=0, \\
\int_{\Omega} \tilde{\mathbf{N}}^{T}\left[L_{1}(\tilde{u})+C_{1}\right] \mathrm{d} \Omega=0 .
\end{gathered}
$$


The first item in (3.10) is the approximate equation of the natural variation of the linear system of equations:

$$
\mathbf{K a}=\mathbf{P} .
$$

Thus, (3.10) and (3.11) could be rewritten in the following form:

$$
\mathbf{K}_{0} \mathbf{C}+\mathbf{R}=\left[\begin{array}{cc}
\mathbf{K} & \mathbf{G} \\
\mathbf{G}^{T} & 0
\end{array}\right]\left\{\begin{array}{l}
\mathbf{a} \\
\mathbf{b}
\end{array}\right\}-\left\{\begin{array}{l}
\mathbf{P} \\
\mathbf{Q}
\end{array}\right\}=0
$$

where

$$
\begin{aligned}
& \mathbf{K}=\int_{\Omega} \mathbf{N}^{T} A(\mathbf{N}) \mathrm{d} \Omega, \\
& \mathbf{G}^{\mathrm{T}}=\int_{\Omega} \tilde{\mathbf{N}}^{T} L_{1}(\mathbf{N}) \mathrm{d} \Omega, \\
& \mathbf{Q}=\int_{\Omega} \tilde{\mathbf{N}}^{T} C_{1} \mathrm{~d} \Omega .
\end{aligned}
$$

\subsection{Construction of quasi-Shannon interval wavelet}

Considering a one-dimensional problem $u(x)$, whose domain of definition $\Omega$ is $[m, n]$. Assuming that the number of discrete point is $2^{j}+1, j \in Z$, the $i$ th discrete point of variable $x$ could be written as

$$
x_{i}=m+\frac{n-m}{2^{j}} \cdot i, \quad i \in Z .
$$

If the quasi-Shannon wavelet with interpolation property is employed as the trial function $N_{j}$, that is,

$$
N_{j}=w_{j}\left(x-x_{i}\right)=\frac{\sin \left(2^{j} \pi /(n-m)\right)\left(x-x_{i}\right)}{\left(2^{j} \pi /(n-m)\right)\left(x-x_{i}\right)} \exp \left(-\frac{2^{2 j-1}\left(x-x_{i}\right)^{2}}{r^{2}(n-m)^{2}}\right) .
$$

Any element in the matrices $\mathbf{K}$ and $G^{T}$ could be expressed, respectively, as

$$
\begin{gathered}
K_{k, n}=A\left(w_{j}(k-n)\right), \\
G_{k, n}^{\mathrm{T}}=\int_{\Omega} \widetilde{N}_{k}^{\mathrm{T}} L_{1}\left(N_{n}\right) \mathrm{d} \Omega,
\end{gathered}
$$

where $\widetilde{N}_{k}$ is the Lagrange basic function.

As the definition domain of wavelet transformation is a double infinite interval, the wavelet coefficients are large near the endpoints of the bounded signal, which increases the 
computational error. To obtain a high computational precision, the additional condition of scalar fonctionelle in boundary $\Gamma$ could be changed to

$$
u=\bar{u}
$$

Thus,

$$
L_{1}\left(N_{n}\right)=\sum_{i} N_{i} \bar{u}_{i}
$$

where $\bar{u}_{i}$ is a known value. Recomputing (3.13) with this additional condition, it could be rewritten as follows:

$$
\mathbf{K}_{0} \mathbf{C}+\mathbf{R}=\left[\begin{array}{cc}
\mathbf{K} & \mathrm{G} \\
\mathrm{G}^{\mathrm{T}} & \mathbf{0}
\end{array}\right]\left\{\begin{array}{l}
\mathbf{a} \\
\mathbf{b}
\end{array}\right\}-\left\{\begin{array}{l}
\mathbf{P} \\
\mathbf{0}
\end{array}\right\}=0
$$

Correspondingly, (3.18) changed to the form

$$
G_{k, n}^{\mathrm{T}}=\widetilde{N}_{k}^{\mathrm{T}} N_{n}
$$

The interpolation properties of $\tilde{\mathbf{N}}$ and $\mathbf{N}$ imply that $\mathbf{a}$ and $\mathbf{b}$ are the vectors consisting of the approximate values of $u$ in discrete points $x_{i}$ and extended discrete points outside the domain of $[m, n]$, respectively. Typically, periodic, symmetry, and zero extension methods could be employed. Assuming that there are $R=2^{j}+1$ discrete points in $[m, n]$, that is, $x_{0}, x_{1}, \ldots, x_{2^{j}}$, and $L$ extended points in both outsides of the domain $[m, n]$, respectively, that is, $x_{-L-1}, x_{-L}, \ldots, x_{-1}$ and $x_{R}, x_{R+1}, \ldots, x_{R+L}$, the function $u$ can be extended outside of the definition domain using the symmetry method, period method, or the zero extension method. Here, we take the symmetry method as example to illustrate the interval wavelet construction method as follows:

$$
\begin{aligned}
u\left(x_{-1}\right) & =u\left(x_{0}\right) \\
u\left(x_{-2}\right) & =u\left(x_{1}\right) \\
& \vdots \\
u\left(x_{-1-i}\right) & =u\left(x_{i}\right) \\
& \vdots \\
u\left(x_{-L-1}\right) & =u\left(x_{L}\right), \\
u\left(x_{R}\right) & =u\left(x_{R-1}\right) \\
u\left(x_{R+1}\right) & =u\left(x_{R-2}\right) \\
& \vdots \\
u\left(x_{R+i}\right) & =u\left(x_{R-i-1}\right) \\
& \vdots \\
u\left(x_{R+L}\right) & =u\left(x_{R-L-1}\right)
\end{aligned}
$$


Substituting (3.23) into (3.21) results in

$$
\left[\begin{array}{lll}
\mathbf{G} 1 & & \\
& \mathbf{A} & \\
& & \mathbf{G} 2
\end{array}\right]\left\{\begin{array}{c}
u_{-L-1} \\
u_{-L} \\
\vdots \\
u_{-1} \\
u_{0} \\
u_{1} \\
\vdots \\
u_{2^{j}} \\
u_{R} \\
u_{R+1} \\
\vdots \\
u_{R+L}
\end{array}\right\}=\left\{\begin{array}{c}
\mathbf{P}^{\prime} \\
\mathbf{0}
\end{array}\right\},
$$

where

$$
\begin{gathered}
\mathbf{A}=\left[\begin{array}{cccc}
A\left(w_{j}(0-0)\right) & A\left(w_{j}(0-1)\right) & \cdots & A\left(w_{j}\left(0-2^{j}\right)\right) \\
A\left(w_{j}(1-0)\right) & A\left(w_{j}(1-1)\right) & \cdots & A\left(w_{j}\left(1-2^{j}\right)\right) \\
\vdots & \vdots & \vdots & \vdots \\
A\left(w_{j}\left(2^{j}-0\right)\right) & A\left(w_{j}\left(2^{j}-1\right)\right) & \cdots & A\left(w_{j}\left(2^{j}-2^{j}\right)\right)
\end{array}\right], \\
\mathbf{G 1}=\left[\begin{array}{cccc}
a_{-L-1,-L-1} w_{j}(0) & a_{-L-1,-L} w_{j}(-1) & \cdots & a_{-L-1,-1} w_{j}(-L) \\
a_{-L,-L-1} w_{j}(1) & a_{-L,-L} w_{j}(0) & \cdots & a_{-L,-1} w_{j}(1-L) \\
\vdots & \vdots & \vdots & \vdots \\
a_{-1,-L-1} w_{j}(L) & a_{-1,-L} w_{j}(L-1) & \cdots & a_{-1,-1} w_{j}(0)
\end{array}\right], \\
\mathbf{G 2}=\left[\begin{array}{cccc}
b_{R, R} w_{j}(0) & b_{R, R+1} w_{j}(-1) & \cdots & b_{R, R+L} w_{j}(-L) \\
b_{R+1, R} w_{j}(1) & b_{R+1, R+1} w_{j}(0) & \cdots & b_{R+1, R} w_{j}(1-L) \\
\vdots & \vdots & \vdots & \vdots \\
b_{R+L, R} w_{j}(L) & b_{R+L, R+1} w_{j}(L-1) & \cdots & b_{R+L, R+L} w_{j}(0)
\end{array}\right] .
\end{gathered}
$$

In the matrices $\mathrm{G} 1$ and $\mathrm{G} 2, a_{n k}$ and $b_{n k}$ could be calculated, respectively, as

$$
\begin{gathered}
a_{n k}=l_{j k}^{1}\left(x_{j n}\right), \quad b_{n k}=l_{j k}^{2}\left(x_{j n}\right), \\
l_{j, k}^{1}=\prod_{\substack{i=-L-1 \\
i \neq k}}^{-1} \frac{x-x_{j, i}}{x_{j, k}-x_{j, i}}, \quad l_{j, k}^{2}=\prod_{\substack{i=2^{j}+1 \\
i \neq k}}^{2^{j}+1+L} \frac{x-x_{j, i}}{x_{j, k}-x_{j, i}} .
\end{gathered}
$$


According to $G^{T} b=0$ and the relational expression (3.23), (3.24) could be condensed in the following way:

$$
\left[\begin{array}{ccc}
\mathrm{G} 1+\mathbf{A} 11 & \mathbf{A} 12 & \mathbf{A} 13 \\
\mathbf{A} 21 & \mathbf{A} 22 & \mathbf{A} 23 \\
\mathbf{A} 31 & \mathbf{A} 32 & \mathbf{G} 2+\mathbf{A} 33
\end{array}\right]\left\{\begin{array}{c}
u_{0} \\
u_{1} \\
\vdots \\
u_{2^{j}}
\end{array}\right\}=\left\{\begin{array}{c}
\mathbf{P} \\
0
\end{array}\right\} .
$$

Subsequently, as interval interpolation basic functions, the quasi-Shannon interval wavelet can be obtained from (3.28) as follows:

$$
\begin{aligned}
& w_{j k}=w\left(2^{j} x-k\right)+\sum_{n=-N+1}^{-1} a_{n k} w\left(2^{j} x-n\right), \quad k=0, \ldots, L, \\
& w_{j k}=w\left(2^{j} x-k\right), \quad k=L+1, \ldots, 2^{j}-L-1, \\
& w_{j k}=w\left(2^{j} x-k\right)+\sum_{n=2^{j}+1}^{2^{j}+N-1} b_{n k} w\left(2^{j} x-n\right), \quad k=2^{j}-L, \ldots, 2^{j},
\end{aligned}
$$

where $L$ is the number of the external points, $N$ is the support domain of the wavelet function, that is, $\sup \phi=[-N, N]$.

It is easy to know that the quasi-Shannon interval wavelet is a linear combination of the quasi-Shannon scaling function $w_{j, k}(x)$. Therefore, the quasi-Shannon interval wavelet function possesses all the properties of the quasi-Shannon scaling function.

It should be noted that the interval wavelet function (3.29) is similar to the result obtained in [5] using different methods. But we can construct different interval wavelets possessed similar format based on different extension technologies by this method. Otherwise, this method reveals the close relationship between the restricted variational principle and the interval interpolation wavelet.

\section{Interval wavelet numerical method for ordinary deferential equations (ODEs)}

\subsection{Interval wavelet discrete formulation of ODEs [6, 7]}

Considering the following ODE:

$$
A(x) u^{\prime \prime}(x)+B(x) u^{\prime}(x)+C(x) u(x)=f(x), \quad x \in[a, b] .
$$

According to the collocation method, its approximate solution can be expressed as

$$
u_{j}(x)=\sum_{n=0}^{2^{j}} u_{j}\left(x_{n}\right) w\left(2^{j} x-n\right), \quad n=0,1,2, \ldots, 2^{j} .
$$

Substituting (4.2) into (4.1), the system of algebraic equations can be obtained as follows:

$$
\begin{array}{r}
\sum_{n=0}^{2^{j}} u_{j}\left(x_{n}\right)\left[A\left(x_{k}\right) 2^{2 j} w^{\prime \prime}(k-n)+B\left(x_{k}\right) 2^{j} w^{\prime}(k-n)+C\left(x_{k}\right) w(k-n)\right]=f\left(x_{k}\right), \\
k=0,1,2, \ldots, 2^{j} .
\end{array}
$$

The solution of (4.3) is an approximate one of (4.1) at the collocation point $x_{k}$. Equation (4.3) could be rewritten in the new form below as follows:

$$
\mathbf{M U}=\mathbf{F},
$$


where $\mathbf{U}$ is the vector of solution, $\mathbf{F}$ is right side vector, and $\mathbf{M}$ is the stiffness matrix. Any element of the matrix $\mathbf{M}$ could be expressed as follows:

$$
m_{k, n}=A\left(x_{k}\right) 2^{2 j} w^{\prime \prime}(k-n)+B\left(x_{k}\right) 2^{j} w^{\prime}(k-n)+C\left(x_{k}\right) w(k-n),
$$

where

$$
\begin{aligned}
& w^{\prime}(k-n)= \begin{cases}\frac{2^{j} \cos [\pi(k-n)] \exp \left[-(k-n)^{2} /\left(2 r^{2}\right)\right]}{(k-n)(b-a)}, & k \neq n \\
0, & k=n,\end{cases} \\
& w^{\prime \prime}(k-n)= \begin{cases}\left.-\frac{2^{2 j+1} \cos [\pi(k-n)] \exp \left[-(k-n)^{2} / 2 r^{2}\right]}{(k-n)^{2}}+\frac{1}{r^{2}}\right], & k \neq n \\
-\frac{2^{2 j}\left(3+\pi^{2} r^{2}\right)}{3 r^{2}(b-a)^{2}}, & (b-a)^{2}\end{cases}
\end{aligned}
$$

In the solution of the above differential equations, there are several types of boundary conditions commonly encountered, such as Dirichlet and Neumann boundary conditions. For simple, we only consider the Dirichlet boundary in the following discussions.

Dirichlet boundary conditions of differential equations specify the value of function on a surface $S$ :

$$
\left.u(x)\right|_{S}=\beta(x), \quad x \in S .
$$

That is, the solution $u(x)$ on the boundary $S$ is known. Assuming that the definition interval of the solution is $[0,1]$, the approximate solution will be

$$
u_{j}(x)=u_{j}(0) w\left(2^{j} x\right)+\sum_{n=1}^{2^{j}-1} u_{j}\left(x_{n}\right) w\left(2^{j} x-n\right)+u_{j}(1) w\left(2^{j} x-2^{j}\right) .
$$

Substituting (4.8) into (4.4), the system of algebraic equations satisfying the boundary conditions could be obtained.

\subsection{Numerical example}

Considering the following two-point boundary problem as a convection equation,

$$
\begin{gathered}
-\varepsilon u^{\prime \prime}(x)+u^{\prime}(x)+u(x)=1, \quad 0<x<1, \\
u(0)=u(1)=0 .
\end{gathered}
$$

Its analytical solution as shown in Figure 1 is

$$
u(x)=\frac{1-e^{\lambda_{2}}}{e^{\lambda_{2}}-e^{\lambda_{1}}} e^{\lambda_{1} x}-\frac{1-e^{\lambda_{1}}}{e^{\lambda_{2}}-e^{\lambda_{1}}} e^{\lambda_{2} x}+1,
$$

where $\lambda_{1}$ and $\lambda_{2}$ are Eigenvalues:

$$
\lambda_{1}=\frac{1+\sqrt{1+4 \varepsilon}}{2 \varepsilon}, \quad \lambda_{2}=\frac{1-\sqrt{1+4 \varepsilon}}{2 \varepsilon} .
$$






Figure 1: Analytical solution of the convection (4.9).



(a) Shannon wavelet



(d)



(b) Quasi-Shannon wavelet



(e)



(c) Interval wavelet

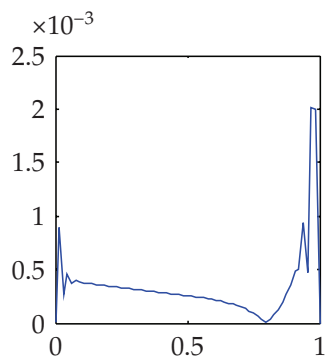

(f)

Figure 2: The comparison of the Shannon (a), the quasi-Shannon (b), and the interval wavelet (c), and their errors of numerical results (e), (f), and (g), respectively.

Obviously, the boundary layer phenomenon is evident near the boundary $x=1$, which would increase with the decrease of the parameter $\varepsilon$. This could be used to test the effectiveness of the interval wavelet.

Substituting (4.5) into (4.9) results in its wavelet discrete formulation as the following:

$$
\sum_{n=1}^{2^{j}-1} u_{j}^{*}\left(x_{n}\right)\left[-2^{2 j} \varepsilon w^{\prime \prime}(k-n)+2^{j} w^{\prime}(k-n)+w(k-n)\right]=1, \quad k=1,2, \ldots, 2^{j}-1
$$


Table 1: Influence of the total number of external collocation points $L(j=6, \varepsilon=1)$.

\begin{tabular}{lcccr}
\hline$L$ & \multicolumn{2}{c}{ Maximum of absolute error $\left(10^{-3}\right)$} & \multicolumn{2}{c}{ Maximum of relative error $\left(10^{-1}\right)$} \\
& Quasi wavelet & Wavelet & Quasi wavelet & Wavelet \\
\hline 1 & 0.293909 & 0.231154 & 0.396774 & 0.214034 \\
2 & 0.501539 & 2.824444 & 0.612304 & 3.448221 \\
3 & 1.257581 & 2.641409 & 1.535317 & 3.855991 \\
4 & 2.080191 & 2.020558 & 2.539601 & 1.273226 \\
\hline
\end{tabular}

The weight function $w$ in (4.12) can be set as the Shannon, the quasi-Shannon, or the interval wavelet functions. Their numerical solutions and corresponding errors are shown in Figure 2, where the count of collocation points is 65 , and the points are distributed evenly in space. Obviously, the calculation precision of the interval wavelet is higher than others.

Besides, the regularized width parameter $\sigma$ which has been discussed in [8], the parameters $L, \varepsilon$, and $j$ affect the calculation precision significantly. Therefore, appropriate values should be selected when implementing the numerical method developed.

\subsubsection{Total number of external collocation points $L$}

In fact, the definition of interval wavelet in (3.29) indicates that the values of $u$ in external collocation points are obtained by Lagrange interpolation method. So, the parameter $L$ is relative with the smoothness of the solution, which is difficult to be determined. With the increasing of $L$, the Runge phenomena could occur in the Lagrange interpolation [9]. As a result, it could decrease the computational precision, which is proved by the numerical results shown in Table 1. When $L=1$, the maxima of both the relative error and the absolute error are apparently less than that of the other two methods. However, opposite results were obtained, when $L=3$. Actually, the solution of ODE with the Dirichlet boundary condition is not smooth on the boundary in most cases, and so the bigger $L$ cannot make any contribution to improve the computational precision except increasing the time complexity of the numerical method. Consequently, $L=1 \sim 3$ might be the best choice.

\subsubsection{Parameter $\varepsilon$}

Table 2 shows the influence of parameter $\varepsilon$ on the calculation precision. It can be observed that the solution is smooth as $\varepsilon=1$. Although the quasi-Shannon wavelet has local property, its computational precision is lower than that of the Shannon wavelet without local property. With $\varepsilon$ decreasing, the superiority of quasi-Shannon wavelet method to Shannon wavelet method exhibits gradually. When $\varepsilon=0.001$, the maximum of the relative error of the quasi-Shannon wavelet method is only 0.33 , while that of Shannon wavelet method is as high as 26.6. The precision of the interval wavelet method keeps higher than the other two methods in the whole process. When $j=6$ and $\varepsilon \geq 0.1$, the maxima of both the relative error and the absolute error of the interval wavelet method are the lowest among the three methods.

However, when the parameter $\varepsilon$ further decreases, the relative error of the interval wavelet method is slightly larger than that of quasi-Shannon wavelet method, and the absolute error of the interval wavelet method approximately identical to that of quasi-Shannon wavelet 
Table 2: Influence of parameter $\varepsilon$.

\begin{tabular}{|c|c|c|c|c|c|c|c|}
\hline \multirow{2}{*}{$j$} & \multirow{2}{*}{$\varepsilon$} & \multicolumn{3}{|c|}{ Maximum of absolute error $\left(10^{-3}\right)$} & \multicolumn{3}{|c|}{ Maximum of relative error $\left(10^{-1}\right)$} \\
\hline & & Wavelet & Quasi wavelet & Interval wavelet & Wavelet & Quasi wavelet & Interval wavelet \\
\hline \multirow{5}{*}{6} & 10 & 0.106914 & 0.111678 & 0.028917 & 1.380331 & 1.441719 & 0.376946 \\
\hline & 1 & 1.123656 & 1.174248 & 0.293909 & 1.378319 & 1.439217 & 0.396774 \\
\hline & 0.1 & 11.376105 & 11.941366 & 2.010026 & 1.420279 & 1.483418 & 0.624155 \\
\hline & 0.01 & 222.141128 & 23.408347 & 57.468784 & 5.043318 & 1.972964 & 1.887983 \\
\hline & 0.001 & 412.488802 & 207.321583 & 411.521362 & 266.324372 & 3.312058 & 6.574245 \\
\hline \multirow{5}{*}{7} & 10 & 0.053260 & 0.056518 & 0.014050 & 1.363706 & 1.424885 & 0.361576 \\
\hline & 1 & 0.563071 & 0.588491 & 0.145969 & 1.362734 & 1.423654 & 0.371681 \\
\hline & 0.1 & 6.085202 & 6.374630 & 1.322155 & 1.386066 & 1.446475 & 0.491532 \\
\hline & 0.01 & 31.345841 & 33.297824 & 1.565785 & 1.347694 & 1.740275 & 1.362828 \\
\hline & 0.001 & 164.839419 & 151.457045 & 33.225063 & 185.349515 & 2.552534 & 5.285445 \\
\hline \multirow{5}{*}{8} & 10 & 0.026582 & 0.027779 & 0.006915 & 1.355528 & 1.4165667 & 0.353550 \\
\hline & 1 & 0.281860 & 0.294593 & 0.072672 & 1.355051 & 1.4159566 & 0.358653 \\
\hline & 0.1 & 3.146638 & 3.292653 & 0.745793 & 1.367255 & 1.427546 & 0.420238 \\
\hline & 0.01 & 23.224318 & 24.535314 & 7.964710 & 1.474450 & 1.588004 & 0.947608 \\
\hline & 0.001 & 56.195135 & 63.509684 & 206.247916 & 57.747668 & 2.338209 & 3.339211 \\
\hline 10 & 0.001 & 31.438062 & 33.344138 & 23.764024 & 0.798518 & 1.807492 & 1.523272 \\
\hline
\end{tabular}

Table 3: Influence of $j(\varepsilon=0.001)$.

\begin{tabular}{lcccccc}
\hline$j$ & \multicolumn{2}{c}{ Maximum of absolute error $\left(10^{-3}\right)$} & \multicolumn{3}{c}{ Maximum of relative error $\left(10^{-1}\right)$} \\
& Wavelet & Quasi wavelet & Interval wavelet & Wavelet & Quasi wavelet & Interval wavelet \\
\hline 6 & 412.488802 & 207.321583 & 411.521362 & 266.324372 & 3.312058 & 6.574245 \\
7 & 164.839419 & 151.457045 & 33.225063 & 185.349515 & 2.552534 & 5.285445 \\
8 & 56.195135 & 63.509684 & 206.247916 & 57.747668 & 2.338209 & 3.339211 \\
10 & 31.438062 & 33.344138 & 23.764024 & 0.798518 & 1.807492 & 1.523272 \\
\hline
\end{tabular}

method. This is due to the boundary layer phenomena of the two-point boundary problem appearing at the points adjacent to the boundary $x=1$. The boundary layer phenomena result in the large gradient of solution, and thus the Gibbs phenomena [10] are unavoidable. Since the construction of the interval wavelet is based on the Lagrange continuation, the Gibbs phenomena would decrease the precision of continuation. Hence, the computational precision of quasi-Shannon interval wavelet method is decreased.

\subsubsection{Influence of $j$}

The parameter $j$ is used to control the total number of collocation points in the discrete formula of wavelet collocation method. The number of collocation points is the exponential function of 
Table 4: Comparison of calculation precision obtained by Shannon and quasi-Shannon interval wavelet methods.

\begin{tabular}{|c|c|c|c|c|c|}
\hline \multirow{2}{*}{$j$} & \multirow{2}{*}{$\varepsilon$} & \multicolumn{2}{|c|}{ Maximum of absolute error $\left(10^{-5}\right)$} & \multicolumn{2}{|c|}{ Maximu of relative error } \\
\hline & & $\begin{array}{l}\text { Shannon Inter- } \\
\text { val wavelet }\end{array}$ & $\begin{array}{l}\text { Quasi-Shannon } \\
\text { Interval wavelet }\end{array}$ & $\begin{array}{l}\text { Shannon Inter- } \\
\text { val wavelet }\end{array}$ & $\begin{array}{l}\text { Quasi-Shannon } \\
\text { Interval wavelet }\end{array}$ \\
\hline \multirow{3}{*}{6} & 10 & 1.957398258 & 2.891724995 & 0.01764588484 & 0.0376945532 \\
\hline & 1 & 23.115408165 & 29.390905441 & 0.02140336631 & 0.0396773857 \\
\hline & 0.1 & 465.352264175 & 201.002620554 & 0.06886365066 & 0.0624155254 \\
\hline \multirow{4}{*}{7} & 10 & 0.900334503 & 1.405000206 & 0.01873761840 & 0.0361575879 \\
\hline & 1 & 1.017560446 & 1.459691836 & 0.02059129393 & 0.0371681364 \\
\hline & 0.1 & 175.721016219 & 132.215467365 & 0.04324788942 & 0.0491531901 \\
\hline & 0.01 & 6008.124739931 & 1565.785030594 & 0.67380962016 & 0.1362827615 \\
\hline 10 & 0.01 & 221.30267 & 150.79668 & 0.05825734364 & 0.0519607606 \\
\hline
\end{tabular}

the parameter $j$, that is, $2^{j}+1$. According to the explanation in Section 4.2.2, if there is no Gibbs phenomena or the Gibbs phenomena are weak enough, the precision of the interval wavelet method should be slightly higher than the quasi-Shannon wavelet method, even the value of $\varepsilon$ is small. The Gibbs phenomena would be continuously weakening up to none with the increment of the collocation points. Table 3 indicates that calculation errors of all three methods decrease with number of the collocation points increasing. When $j=10$, the maximum absolute error of the interval wavelet method is less than the other two methods. In addition, the maximum relative error of the interval wavelet method is less than the error of the quasi-Shannon wavelet method, but larger than the error of Shannon wavelet method.

\subsection{Comparison of calculation precision obtained by Shannon interval wavelet method and quasi-Shannon interval wavelet method}

In this work, the Shannon interval wavelet is also constructed to further evaluate the performance of the quasi-Shannon interval wavelet for numerical analysis. Instead of (3.16), the following weight function is used to construct the Shannon interval wavelet:

$$
w_{j}\left(x-x_{i}\right)=\frac{\sin \left[\left(2^{j} \pi /(b-a)\right)\left(x-x_{i}\right)\right]}{\left(2^{j} \pi /(b-a)\right)\left(x-x_{i}\right)} .
$$

The first and second derivatives of (4.13) are expressed, respectively, as follows:

$$
\begin{aligned}
& w_{j}^{\prime}\left(x-x_{i}\right)=\left\{\begin{array}{lr}
\frac{\cos \left[\left(2^{j} \pi /(b-a)\right)\left(x-x_{i}\right)\right]}{x-x_{i}}-\frac{b-a}{2^{j} \pi} \cdot \frac{\sin \left[\left(2^{j} \pi /(b-a)\right)\left(x-x_{i}\right)\right]}{\left(x-x_{i}\right)^{2}}, & x \neq x_{i}, \\
0, & x=x_{i},
\end{array}\right. \\
& w_{j}^{\prime \prime}\left(x-x_{i}\right)= \begin{cases}\frac{2 \sin \left[\left(2^{j} \pi\left(x-x_{i}\right) /(b-a)\right)\right]}{2^{j} \pi\left(x-x_{i}\right)^{3} /(b-a)}-\frac{2 \cos \left[\left(2^{j} \pi\left(x-x_{i}\right) /(b-a)\right)\right]}{\left(x-x_{i}\right)^{2}} & x \neq x_{i}, \\
-\frac{2^{j} \pi \sin \left[\left(2^{j} \pi\left(x-x_{i}\right) /(b-a)\right)\right]}{(b-a)\left(x-x_{i}\right)}, & x=x_{i} . \\
-\frac{2^{2 j} \pi^{2}}{3(b-a)^{2}}, & \end{cases}
\end{aligned}
$$






(a) quasi-Shannon interval wavelet



(b) Shannon interval wavelet

Figure 3: Comparison of the quasi-Shannon interval wavelet and the Shannon interval wavelet $(j=7$, $\varepsilon=0.4$, and $L=1$ ).

The collocation method of Shannon interval wavelet method could be obtained by substituting (4.13)-(4.15) into (4.5).

Table 4 indicates that the maxima of both the relative error and the absolute error obtained by the Shannon interval wavelet method are less than those obtained by the quasiShannon interval wavelet method when the solution curve of the two-point problem is smooth. As $\varepsilon$ decreasing, the precision of quasi-Shannon interval method become higher than that of the Shannon interval wavelet method. Especially when $\varepsilon \leq 0.01$, the calculation precision obtained by quasi-Shannon interval wavelet method is higher than that obtained by Shannon interval wavelet method in the whole process with the collocation number varying from a smaller number to 1025 (i.e., $j=10$ ).

Figure 3 also shows that when the equation is solved by the quasi-Shannon interval wavelet, the numerical error mainly lies in the boundary, while the errors of other parts are very little. On the contrast, when the Shannon interval wavelet method is utilized, the numerical error almost evenly distributes in the all domain. Consequently, the sum of the error is much larger than that of the quasi-Shannon interval wavelet.

\section{Conclusions}

A novel general method for construction of interval wavelet based on the restricted variational principle is proposed in this paper. Both Shannon and quasi-Shannon interval wavelets could be constructed using this new method. With appropriate values of parameters $L, \varepsilon$, and $j$ selected, the numerical results for a differential equation show that the quasi-Shannon interval wavelet outperforms than Shannon and quasi-Shannon wavelets. Furthermore, the capability of quasi-Shannon interval wavelet for numerical analysis is better than that of Shannon interval wavelet.

It should be noted that combining the corresponding interval wavelet with the classical Galerkin method can obtain an excellent wavelet-Galerkin method for engineering problems. Since quasi-Shannon wavelet is orthogonal, this guarantees the stability of the matrix equations in Galerkin method. 


\section{Acknowledgment}

This work is supported by the National Natural Science Foundation of China (no. 60772038).

\section{References}

[1] A. Cohen, I. Daubechies, and P. Vial, "Wavelets on the interval and fast wavelet transforms," Applied and Computational Harmonic Analysis, vol. 1, no. 1, pp. 54-81, 1993.

[2] E. Quak and N. Weyrich, "Decomposition and reconstruction algorithms for spline wavelets on a bounded interval," Applied and Computational Harmonic Analysis, vol. 1, no. 3, pp. 217-231, 1994.

[3] C. R. Ortloff, "Restricted variational principle methods for boundary value problems of kinetic theory," Physics of Fluids, vol. 10, no. 1, pp. 230-231, 1967.

[4] G. W. Wei, "Quasi wavelet and quasi interpolating wavelets," Chemical Physics Letters, vol. 296, no. 3-4, pp. 215-222, 1998.

[5] O. V. Vasilyev and S. Paolucci, "A dynamically adaptive multilevel wavelet collocation method for solving partial differential equations in a finite domain," Journal of Computational Physics, vol. 125, no. 2, pp. 498-512, 1996.

[6] S.-L. Mei, Q.-S. Lu, S.-W. Zhang, and L. Jin, "Adaptive interval wavelet precise integration method for partial differential equations," Applied Mathematics and Mechanics, vol. 26, no. 3, pp. 364-371, 2005.

[7] S.-L. Mei, Q.-S. Lu, and S.-W. Zhang, "An adaptive wavelet precise integration method for partial differential equations," Chinese Journal of Computational Physics, vol. 21, no. 6, pp. 523-530, 2004.

[8] D.-C. Wan and G.-W. Wei, "The study of quasi wavelets based numerical method applied to Burgers' equations," Applied Mathematics and Mechanics, vol. 21, no. 10, pp. 1099-1110, 2000.

[9] J. J. Leader, Numerical Analysis and Scientific Computation, Addison-Wesley, Reading, Mass, USA, 2004.

[10] I. Daubechies, Ten Lectures on Wavelets, vol. 61 of CBMS-NSF Regional Conference Series in Applied Mathematics, SIAM, Philadelphia, Pa, USA, 1992. 




Advances in

Operations Research





\section{The Scientific} World Journal


International Journal of

Mathematics and

Mathematical

Sciences
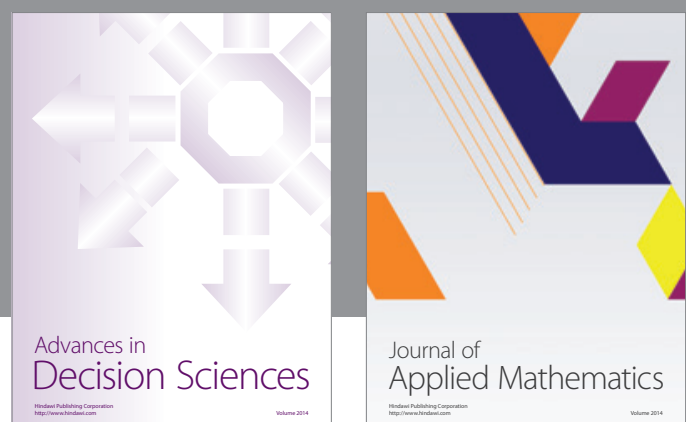

Journal of

Applied Mathematics


Submit your manuscripts at http://www.hindawi.com


Mathematical Problems in Engineering
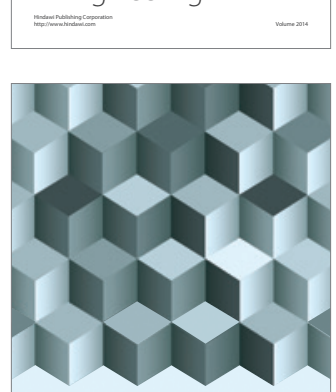

Journal of

Function Spaces


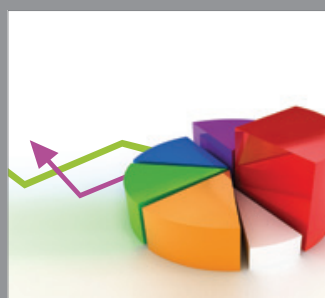

ournal of

Probability and Statistics

Promensencen
